\title{
Combined Amperometric/Field-Effect Sensor for the Detection of Dissolved Hydrogen in Biogas Reactors
}

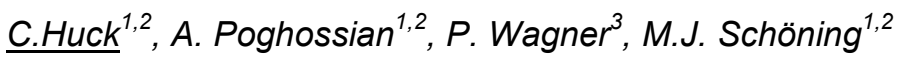 \\ huck@fh-aachen.de \\ ${ }^{1}$ Institute of Nano- and Biotechnologies (INB), Aachen University of Applied Sciences, 52428 Jülich, \\ Germany \\ 2 Peter Grünberg Institute (PGI-8), Research Centre Jülich GmbH, 52525 Jülich, Germany \\ ${ }^{3}$ Institute for Materials Research (IMO), Hasselt University, 3590 Diepenbeek, Belgium
}

\begin{abstract}
:
A one-chip integrated combined amperometric/field-effect sensor for monitoring the dissolved hydrogen $\left(\mathrm{H}_{2}\right)$ concentration has been developed for biogas applications. The combination of two different transducer principles might allow a more accurate and reliable measurement of dissolved $\mathrm{H}_{2}$ as an early warning indicator of digester failures. The proposed approach has been demonstrated by simultaneous amperometric/field-effect measurements of dissolved $\mathrm{H}_{2}$ concentration in electrolyte solutions. Both, the amperometric and the field-effect transducer show a linear response in the $\mathrm{H}_{2}$ concentration range from 0.1 to $3 \% \mathrm{v} / \mathrm{v}$ with a slope of $198.4 \pm 13.7 \mathrm{nA} / \% \mathrm{v} / \mathrm{v}$ and $14.9 \pm$ $0.5 \mathrm{mV} / \% \mathrm{v} / \mathrm{v}$, respectively.
\end{abstract}

Key words: dissolved hydrogen, amperometric gas sensor, field-effect sensor, biogas

\section{Introduction}

The use of biogas as energy source is currently establishing in the group of alternative energies. Accompanied by the growing number of biogas plants, the demand for online monitoring and control of biogas production is increasing [1]. An implementation of chip-based multi-parameter monitoring and control systems could improve the biogas process stability and prevent digester breakdowns. Moreover, process performance for better economy of the biogas plants could be enhanced.

The concentration of dissolved hydrogen $\left(\mathrm{H}_{2}\right)$ represents one of the most important parameters for biogas process control and early warning of process disturbances [2, 3]. Accumulated hydrogen strongly inhibits the degradation of volatile fatty acids, such as propionate and butyrate, resulting in a consequent deterioration of the normal operation [4, 5]. If hydrogen production exceeds the maximum ability of the methanogenic biomass to degrade hydrogen, there will be a rapid and large increase in the hydrogen concentration prior to digester failures. Thus, dissolved $\mathrm{H}_{2}$ is a key factor in the intricate balance between microbial species involved in the multi-step degradation during anaerobic digestion, making it as useful parameter for process monitoring [6].
In this work, a Si-based combined chemosensor capable for the simultaneous amperometric/field-effect detection of the concentration of dissolved $\mathrm{H}_{2}$ has been developed for biogas applications. Such a combination of two different transducer principles for the detection of the same parameter might allow a more accurate, selective and reliable measurement of dissolved $\mathrm{H}_{2}$ as an early warning indicator of digester failures. The functioning of the developed one-chip integrated dual amperometric/field-effect sensor device has been tested in electrolyte solutions with different concentration of dissolved $\mathrm{H}_{2}$.

\section{Structure and functioning principle of the combined $\mathrm{H}_{2}$ sensor}

The schematic layer structure of the combined hydrogen sensor and measurement setup for simultaneous amperometric and field-effect detection of dissolved $\mathrm{H}_{2}$ in electrolyte solutions is shown in Fig. 1. The developed sensor combines a $\mathrm{pH}$-sensitive capacitive electrolyteinsulator-semiconductor (EIS) sensor consisting of an Al-p-Si-SiO $-\mathrm{Ta}_{2} \mathrm{O}_{5}$ structure and a thinfilm $\mathrm{Pt}$ electrode for field-effect and amperometric measurements, respectively. Since the part of the gate region of the fieldeffect sensor is covered with the Pt-metal electrode, it represents a parallel connection of a $\mathrm{pH}$-sensitive capacitive EIS sensor and the 
well-known Pt-gate MIS (metal-insulatorsemiconductor) structure. The latter one is widely used for $\mathrm{H}_{2}$ detection in gaseous atmospheres (see e.g., [7, 8]). As a result, the fieldeffect part of the combined sensor is able to detect dissolved $\mathrm{H}_{2}$ via two mechanisms: a) detection of the product of $\mathrm{H}_{2}$ oxidation with the $\mathrm{pH}$-sensitive sensor, i.e. $\mathrm{H}^{+}$ions generated at the polarized working electrode of the amperometric part of the combined sensor, and b) flatband-voltage variations caused from the formation of hydrogen dipoles at the metalgate/insulator interface of the MIS structure.

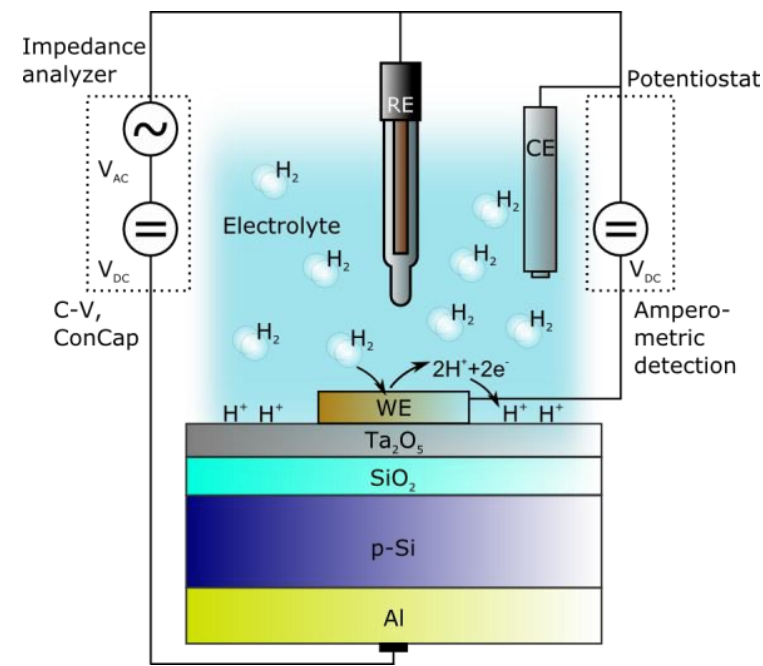

Fig. 1. Schematic layer structure of the combined hydrogen sensor and measurement setup for the simultaneous amperometric/field-effect detection of dissolved hydrogen in electrolyte solutions. WE: Pt working electrode; CE: counter electrode; RE: reference electrode.

\section{Fabrication of the combined sensor structure}

For the fabrication of the combined sensor, capacitive $\mathrm{Al}-\mathrm{Si}-\mathrm{SiO}_{2}-\mathrm{Ta}_{2} \mathrm{O}_{5} \quad(p-\mathrm{Si}, \quad \rho=1-$ $10 \Omega \mathrm{cm} ; \quad 30 \mathrm{~nm}$ thermally grown $\mathrm{SiO}_{2}$ ) structures with $\mathrm{Ta}_{2} \mathrm{O}_{5}$ as $\mathrm{pH}$-sensitive gate insulator have been fabricated. $\mathrm{Ta}_{2} \mathrm{O}_{5}$ is widely used for field-effect $\mathrm{pH}$ sensing, due to its high $\mathrm{pH}$-sensitivity [9] as well as high corrosionresistance properties in a wide $\mathrm{pH}$ range [10]. The $\mathrm{Ta}_{2} \mathrm{O}_{5}$ films were prepared by means of thermal oxidation of an electron-beam deposited $30 \mathrm{~nm}$ Ta layer in a dry oxygen atmosphere at $517^{\circ} \mathrm{C}$ for about $0.5 \mathrm{~h}$, yielding a $\sim 60 \mathrm{~nm}$ thick $\mathrm{Ta}_{2} \mathrm{O}_{5}$ layer. A $300 \mathrm{~nm}$ Al film was deposited as rear-side contact for the fieldeffect sensor. For the realization of the amperometric transducer, a $200 \mathrm{~nm}$ thick platinum electrode was prepared onto a $20 \mathrm{~nm}$ thick titanium adhesion layer by means of electron-beam evaporation and patterned by photolithography and lift-off technique, respec- tively. Finally, the structures were cut into chips with a size of $10 \mathrm{~mm} \times 14 \mathrm{~mm}$ and assembled onto a printed circuit board (PCB). For electrical connection of the EIS structure, the Al rear-side contact was glued with electrically conductive adhesive onto the PCB substrate. The frontside contacts to the thin-film Pt electrodes were provided by means of an ultrasonic wedge bonder.

\section{Gas-sensing setup and testing methodology}

For experiments, the PCB substrate with the combined sensor structure was mounted into a home-made measuring cell and tested in electrolyte solutions with different concentrations of dissolved $\mathrm{H}_{2}$ from 0.05 to $3 \% \mathrm{v} / \mathrm{v}$. The sidewalls and rear-side contact of the combined sensor chip were protected from the electrolyte solution by means of an O-ring, thereby circumventing the need for a complicated encapsulation process. The contact area of the EIS sensor with the solution was about $0.5 \mathrm{~cm}^{2}$.

Hydrogen gas was dissolved in the electrolyte through a perforated tube by mixing with nitrogen in various ratios using commercial massflow controllers (Bronkhorst High-Tech). As electrolyte a $0.25 \mathrm{mM}$ polymix multi-component buffer solution $(\mathrm{pH} 7)$ containing $100 \mathrm{mM} \mathrm{NaCl}$ as an ionic-strength adjuster was used [11, 12]. The $\mathrm{pH}$ of the polymix buffer was adjusted by titration either with $\mathrm{NaOH}$ or $\mathrm{HCl}$.

The combined sensor structure has been characterized by means of amperometric method using a voltage source (Keithley 2400) and constant-capacitance (ConCap) method using an impedance analyzer (Zahner Elektrik) as presented in Fig. 1. For the amperometric detection of dissolved $\mathrm{H}_{2}$ a standard threeelectrode configuration under potential control was used. The potential of the working electrode was set at $+0.55 \mathrm{~V}$ versus the $\mathrm{Ag} / \mathrm{AgCl}$ reference electrode (conventional liquid-junction $\mathrm{Ag} / \mathrm{AgCl}$ electrode, Metrohm) and the hydrogen concentration-dependent current was detected between the working and the counter electrode (Metrohm). The ConCap mode allows dynamic characterization of the EIS structure. In this mode, the capacitance of the EIS sensor is kept constant (usually within the depletion region of the capacitance-voltage curve at $\sim 60 \%$ of the maximum capacitance) using a feedback-control circuit, and potential changes at the transducer/electrolyte interface have been directly monitored. For operation, a DC polarization voltage is applied via the reference electrode to set the working point of the EIS sensor, and a small AC voltage $(20 \mathrm{mV})$ is applied to the system in order to measure the capacitance of the sensor. All measurements 
were carried out at a frequency of $120 \mathrm{~Hz}$ in a dark Faraday cage at room temperature.

\section{Results and discussion}

Before $\mathrm{H}_{2}$ experiments were performed, the $\mathrm{pH}$ sensitivity of the capacitive field-effect Al-p-Si$\mathrm{SiO}_{2}-\mathrm{Ta}_{2} \mathrm{O}_{5}$ structure had been investigated in Titrisol buffers.

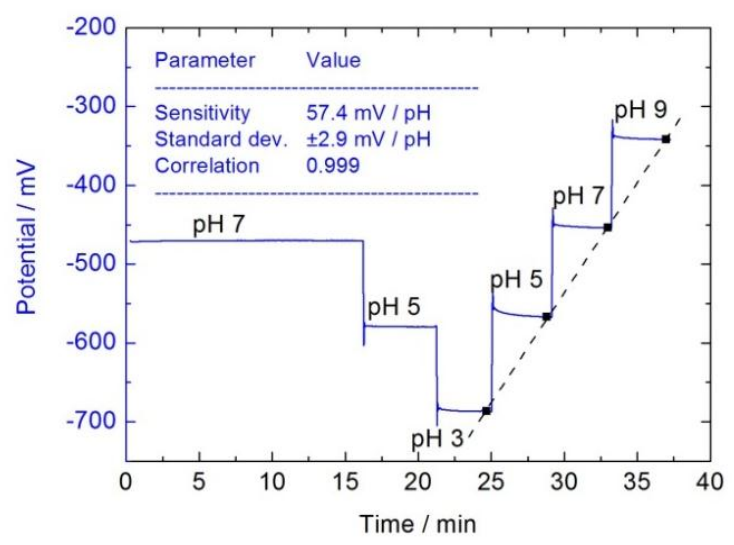

Fig. 2. Typical dynamic ConCap response of the capacitive field-effect Al-p-Si-SiO ${ }_{2}-\mathrm{Ta}_{2} \mathrm{O}_{5} \quad \mathrm{EIS}$ structure recorded in Titrisol buffer with different $\mathrm{pH}$ values from $\mathrm{pH} 3$ to $\mathrm{pH} 9$ and corresponding calibration curve (dashed line).

Figure 2 depicts a typical dynamic ConCap response of the EIS structure recorded in Titrisol buffer solution with different $\mathrm{pH}$ values. The bare EIS sensor shows an average $\mathrm{pH}$ sensitivity of $57.4 \pm 2.9 \mathrm{mV} / \mathrm{pH}$ in the range from $\mathrm{pH} 3$ to 9 , which is in good agreement with $\mathrm{pH}$-sensitivity values reported in literature for a $\mathrm{Ta}_{2} \mathrm{O}_{5}$ layer $[7,9]$.

Figure $3 a$ demonstrates an example of a simultaneous amperometric/field-effect detection of dissolved $\mathrm{H}_{2}$ in the concentration range from 0.05 to $3 \% \mathrm{v} / \mathrm{v} \mathrm{H}_{2}$. With ascending and descending $\mathrm{H}_{2}$ dosages, a good correlation between the ConCap response of the fieldeffect sensor and the output signal of the amperometric sensor has been observed. The respective calibration curves of the combined sensor evaluated from Figure $3 a$ are presented in Figure $3 \mathrm{~b}$. Both, the amperometric and the field-effect transducer show a linear response in the $\mathrm{H}_{2}$ concentration range from 0.1 to $3 \% \mathrm{v} / \mathrm{v}$ with a $\mathrm{H}_{2}$ sensitivity of $198 \pm 14 \mathrm{nA} / \% \mathrm{v} / \mathrm{v}$ and $15 \pm 0.5 \mathrm{mV} / \% \mathrm{v} / \mathrm{v}$, respectively.
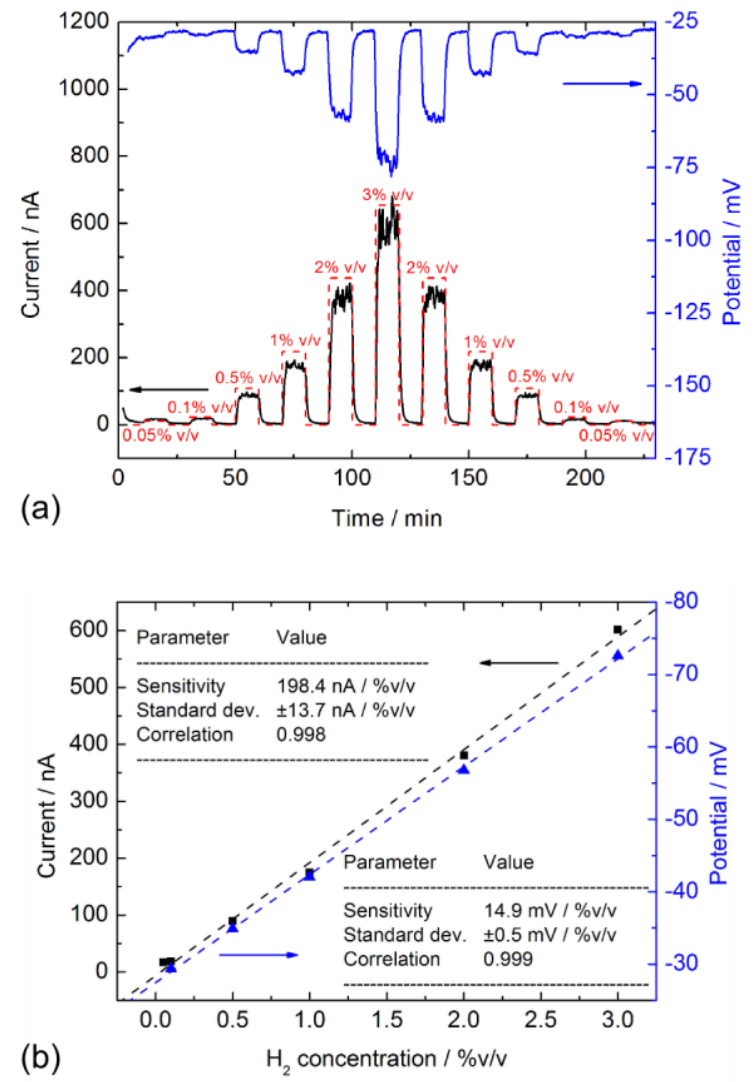

Fig. 3. Simultaneous amperometric/field-effect measurement of dissolved $\mathrm{H}_{2}$ with the combined sensor chip (a) and calibration curves for the fieldeffect and amperometric sensor, respectively (b).

\section{Conclusions}

The obtained experimental results demonstrate the feasibility of the developed combined sensor chip for the simultaneous amperometric/field-effect detection of dissolved $\mathrm{H}_{2}$ in electrolyte solutions. Such a combination of two transducer principles, namely, the amperometric and field-effect detection, might allow in future more accurate, selective and reliable measurements of dissolved $\mathrm{H}_{2}$ in biogas reactors as an early warning indicator of process disturbances or digester failures.

\section{Acknowledgements}

The authors thank the Federal Ministry of Education and Research (BMBF, Germany) for financial support of the project "EMSiG".

\section{References}

[1] P. Weiland, Biogas production: current state and perspectives, Applied Microbiology and Biotechnology 85, 849-860 (2010); doi: $10.1007 / \mathrm{s} 00253-009-2246-7$

[2] D.B. Archer, M.G. Hilton, P. Adams, H. Wiecko, Hydrogen as a process control index in a pilot scale anaerobic digester, Biotechnology Letters 8, 197-202 (1986); doi: 10.1007/BF01029380 
[3] T.N. Whitmore, D. Lloyd, G. Jones, T.N. Williams, Hydrogen-dependent control of the continuous anaerobic digestion process, Applied Microbiology and Biotechnology 26, 383-388 (1987); doi: 10.1007/BF00256675

[4] S.R. Harper, F.G. Pohland, Recent developments in hydrogen management during anaerobic biological wastewater treatment, Biotechnology and Bioengineering 28, 585-602 (1986); doi: 10.1002/bit.260280416

[5] M.J. Mclnerney, M.P. Bryant, Review of methane fermentation fundamentals, in: D.L. Wise (Ed.), Fuel gas production from biomass, CRC Press, Boca Raton, FI., 1981, pp. 20-46.

[6] G.E. Strong, R. Cord-Ruwisch, An in situ dissolved-hydrogen probe for monitoring anaerobic digesters under overload conditions, Biotechnology and Bioengineering 45, 63-68 (1995); doi: 10.1002/bit.260450109

[7] M. Eriksson, A. Salomonsson, I. Lundström, The influence of the insulator surface properties on the hydrogen response of field-effect gas sensors, Journal of Applied Physics 98, 034903 (2005); doi: 10.1063/1.1994941

[8] M. Löfdahl, M. Eriksson, M. Johansson, I. Lundström, Difference in hydrogen sensitivity between $\mathrm{Pt}$ and $\mathrm{Pd}$ field-effect devices, Journal of Applied Physics 91, 4275-4280 (2002); doi: 10.1063/1.1448874

[9] A. Poghossian, The super-nernstian $\mathrm{pH}$ sensitivity of $\mathrm{Ta}_{2} \mathrm{O}_{5}$-gate ISFETs, Sensors and Actuators B 7, 367-370 (1992); doi: 10.1016/0925-4005(92)80326-S

[10] M.J. Schöning, D. Brinkman, D. Rolka, C. Demuth, A. Poghossian, CIP (cleaning-in-place) suitable „non-glass" $\mathrm{pH}$ sensor based on a Ta2O5-gate EIS structure, Sensors and Actuators B 111-112, 423-429 (2005); doi: 10.1016/j.snb.2005.03.053

[11] A.P. Soldatkin, D.V. Gorchkov, C. Martelet, N. Jaffrezic-Renault, Application of charged polymeric materials as additional permselective membranes for modulation of the working characteristics of penicillin sensitive ENFETs, Materials Science and Engineering: C 5, 35-40 (1997); doi: 10.1016/S0928-4931(97)00020-9

[12] T. Yoshinobu, H. Ecken, A. Poghossian, A. Simonis, H. Iwasaki, H. Lüth, M.J. Schöning, Constant-current-mode LAPS (CLAPS) for the detection of penicillin, Electroanalysis 13, 733736 (2001); doi: 10.1002/15214109(200105)13:8/9<733::AIDELAN733>3.0.CO;2-N 